\title{
A Note on VHF Reflection From a Tropospheric Layer
}

\author{
James R. Wait
}

\section{Contribution From the Central Radio Propagation Laboratory, National Bureau of Standards, Boulder Colo.}

(Received March 3, 1964)

\begin{abstract}
Some remarks concerning reflection from tropospheric layers are made with special reference to a recent paper by Bean, Frank, and Lane on the subject. It is indicated that the finite vertical extent of the layer must be considered in the analysis.
\end{abstract}

In a recent interesting paper, Bean, Frank, and Lane [1963] discuss the cumulative probability distribution of field strength for VHF paths in terms of various refractive index profiles. It is claimed that the best agreement is obtained using a layer model with a linear $n$-profile. As indicated in their paper, a typical value of layer thickness is $100 \mathrm{~m}$ with a horizontal dimension of the order of tens of kilometers. The particular layer profile used by Bean et al., has a linear decrease of $n$ over a height interval $h$. Using a method attributed to Brekhovskikh [1960], they state that the reflection coefficient has a magnitude given by

$$
|\boldsymbol{\rho}|=\Delta n \cdot \lambda /\left(8 \pi h \sin ^{3} \alpha\right),
$$

where $\Delta n$ is the total change of $n, \gamma$ is the wavelength, and $\alpha$ is the grazing angle of incidence. However, on close inspection of the matter, it turns out that this result is applicable to an infinite layer which has a gradient numerically equal to $\Delta n / h$. This fact is confirmed by examining the structure of the exact solution of the problem in terms of Bessel functions of order one-third [Wait, 1958].

The correct form of the solution, for the present application, is

$$
|\rho|=\left|\frac{\Delta n}{2 \sin ^{2} \alpha}\left(\frac{\sin \chi}{\chi}\right)\right|
$$

where $\chi=(2 \pi / \lambda) h \sin \alpha$. It may be readily verified that

$$
\operatorname{Lim}_{x \rightarrow 0}|\rho|=\frac{\Delta n}{2 \sin ^{2} \alpha} \simeq \frac{\Delta n}{2 \alpha^{2}}=\rho_{0}
$$

which is the value corresponding to a discontinuity of amount $\Delta n$. Limitations of this type formula have been discussed extensively by the author [Wait, 1962].

To illustrate the significance of the corrected formula, $|\rho|$ in $\mathrm{dB}$ is plotted as a function of $\alpha$ in milliradians in figures 1 and 2. The layer thickness $h$ is $100 \mathrm{~m}$ and the wavelengths chosen are 4.18

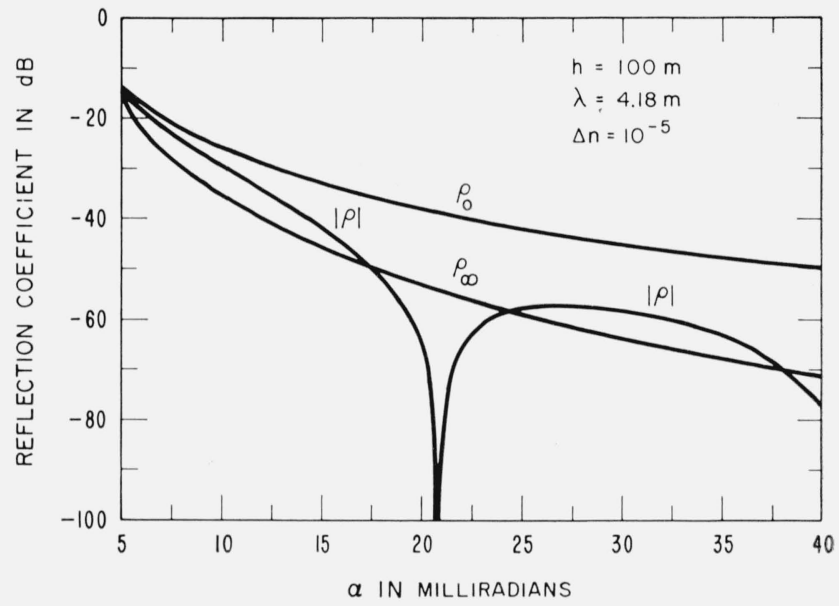

Figure 1. Reflection coefficients at $\lambda=4.18 \mathrm{~m}$ for finite and infinite linear profiles and for a discontinuity of refractive index.

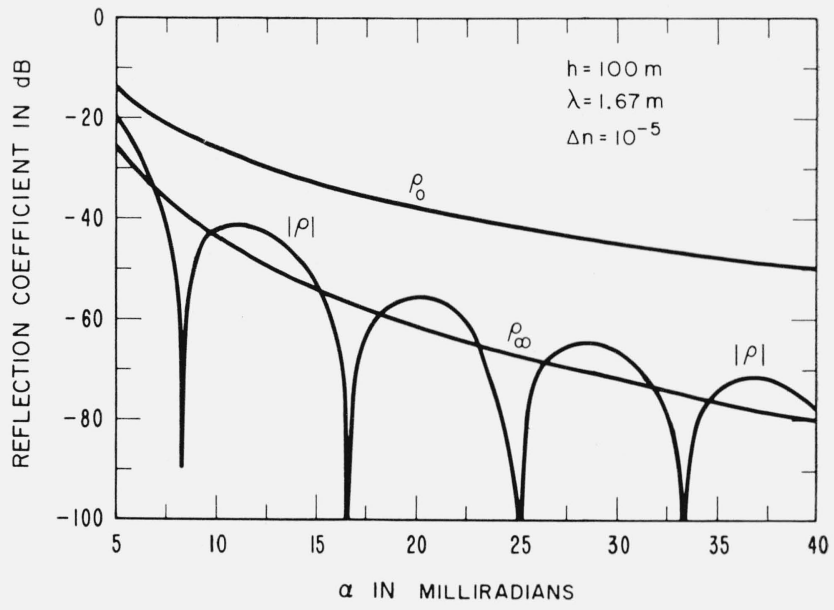

Figure 2. Reflection coefficients at $\lambda=1.67 \mathrm{~m}$ for finite and infinite linear profiles and for a discontinuity of refractive index. 
$\mathrm{m}$ and $1.67 \mathrm{~m}$, corresponding to the examples in the paper by Bean et al. [1963]. For the examples shown in figures 1 and $2, \Delta n$ is taken to be $10^{-5}$, which is also considered typical. Along with the curves for $|\rho|$, the reflection coefficient $\rho_{0}$ for a sharp discontinuity, and the curves for $\rho_{\infty}$, corresponding to Bean's Frank's, and Lane's infinite layer are also shown.

It is evident from the graphical data given here that the angular dependence for the finite linear layer is quite different from the infinite linear layer with the same slope. Of course, both of these reflection coefficients are somewhat below the corresponding value for a discontinuous layer with a change of refractive index of amount $\Delta n$. It is suggested that the oscillatory dependence of $|\rho|$ on the parameter $\chi$ is compatible with the spread of experimental points in figure 5 of the paper by Bean et al. [1963]. It would be worthwhile to make a more systematic study of the radiosonde data with a view to correlating observed fields with the vertical extent of the layer structure.

\section{Reply by J. A. Lane:}

"The authors are indebted to Dr. Wait for drawing" their attention to the limitations of a formula which has been widely used for calculating reflection coefficients of layers with linear gradients of refractive index. We support the suggestion that studies of field strength variation and layer thickness would be valuable."

\section{References}

Bean, B. R., V. R. Frank, and J. A. Lane (Nov.-Dec. 1963), A radio meteorological study, part II. An analysis of VHF field strength variations and refractive index profiles, J. Res. NBS 67D (Radio Prop.), No. 6, 597-604.

Brekhovskikh, L. M. (1960), Waves in layered media (Academic Press, New York and London).

Wait, J. R. (Sept. 1958), Transmission and reflection of electromagnetic waves in the presence of stratified media, J. Res. NBS 61, No. 3, 205-232.

Wait, J. R. (1962), Electromagnetic waves in stratified media, Chapter IV (Pergamon Press, London).

(Paper 68D7-381) 